\title{
OBSERVACIONES SOBRE EL CARANCHO NEGRO PHALCOBOENUS AUSTRALIS (GMELIN, 1788) EN ISLA GONZALO, ARCHIPIELAGO DIEGO RAMÍREZ, CHILE
}

\author{
OBSERVATIONS ON THE STRIATED CARACARA PHALCOBOENUS AUSTRALIS \\ (GMELIN, 1788) IN GONZALO ISLAND, DIEGO RAMIREZ ARCHIPELAGO, CHILE
}

Jaime A. Cursach ${ }^{1,2}$, Cristián G. Suazo ${ }^{3}$, Roberto P. Schlatter ${ }^{4}$ \& Jaime R. Rau ${ }^{2,5}$

El carancho negro Phalcoboenus australis (Gmelin, 1788) es un ave rapaz endémica del sur de la Patagonia, que habita en costas de islas australes expuestas al océano, asociando su presencia a loberías y colonias de aves marinas (Frere et al. 1999, Marín et al. 2006, Catry et al. 2008, BirdLife International 2012). A nivel mundial, el estado de conservación del carancho negro lo clasifica como una especie casi amenazada de extinción, mientras que en Chile conforma el grupo de aves rapaces con máxima prioridad de conservación (Pincheira-Ulbrich et al. 2008, BirdLife International 2012).

La información publicada sobre el carancho negro en Chile es escasa y radica principalmente en el trabajo de Marín et al. (2006), quienes lo describen como localmente abundante en islas exteriores de la región de Magallanes (XII Región), entre los 48 y $56^{\circ}$ $\mathrm{S}$, indicando su nidificación en isla Noir (54 $28^{\circ}$ 'S; $\left.73^{\circ} 00^{\prime} \mathrm{O}\right)$. Mientras que el resto de la información se concentra en el archipiélago Diego Ramírez
(56 $31^{\circ} \mathrm{S}$; 68 $\left.48^{\circ} \mathrm{O}\right)$, lugar que corresponde a su distribución más austral y en el cual aún no existe información detallada sobre su nidificación (Wallace 1991, Schlatter \& Riveros 1997, Marín et al. 2006). Por ello, la presente nota aporta los primeros datos publicados sobre la nidificación del carancho negro en islas Diego Ramírez, sur de Chile, así como también observaciones referentes a su población, dieta y conductas en estas islas.

Este trabajo se realizó en isla Gonzalo (56³1'16,8's; 6842'53,5'O), ubicada en el margen austral del archipiélago Diego Ramírez, sur de Chile. Dicho archipiélago se ubica a $112 \mathrm{~km}$ al suroeste del Cabo de Hornos y representa geográficamente el extremo más austral de América del Sur (Schlatter \& Riveros 1997, Cursach et al. 2012). Las observaciones sobre el carancho negro en isla Gonzalo, integran campañas ornitológicas correspondientes a las temporadas 1980-81, 2010-11 y las realizadas el día 03 de octubre de 2012. Para esta última fecha,

1 Laboratorio de Investigación Socioambiental, Programa ATLAS, Departamento de Ciencias Sociales, Universidad de Los Lagos, casilla 933, Osorno, Chile. jcurval@gmail.com.

2 Laboratorio de Ecología, Departamento de Ciencias Básicas, Universidad de Los Lagos, casilla 933, Osorno, Chile.

3 Albatross Task Force - Chile, Birdlife International.

4 Instituto de Ciencias Marinas y Limnológicas, Facultad de Ciencias, Universidad Austral de Chile. Casilla 567, Valdivia, Chile

5 Programa IBAM, Universidad de Los Lagos, casilla 933, Osorno, Chile. 
se sumaron entrevistas realizadas al personal de la Armada de Chile que cumple tareas de vigilancia permanente en el Faro de isla Gonzalo, a quienes se les consultó sobre temáticas de ecología y apreciaciones personales sobre los caranchos negros que habitan en la isla (Tabla 1). Las mediciones morfológicas realizadas a las aves fueron obtenidas mediante Vernier $( \pm 0,1 \mathrm{~mm})$ y regleta graduada $( \pm$ $0,5 \mathrm{~mm}$ ), mientras que el peso fue registrado con una balanza de resorte ( $\pm 50 \mathrm{~g}$ ).

Durante el verano de 1981 se registró un total de 15 caranchos negros en las islas del grupo sur (isla Gonzalo, isla Bartolomé e islote Ester) de Diego Ramírez, mientras que en las islas del grupo norte (islote Norte e islote Schlatter) se observó la mayor concentración de esta rapaz ( $>40$ individuos). Para el verano 2011, en isla Gonzalo el máximo número de individuos observados en una misma oportunidad (sector rocoso de la zona supra e intermareal) fue de cinco aves, de las cuales tres poseían plumaje de madurez sexual (i.e., abdomen y calzones interiores de color café rojizo en el adulto, mientras que el inmaduro poseía cera y patas de color rosado pálido, con plumaje café en las partes superiores del cuerpo). De forma similar, durante octubre 2012 en isla Gonzalo se observaron nueve individuos en una misma ocasión, de los cuales cinco poseían plumaje de madurez sexual.

Durante el verano de 1981 en la costa noreste de isla Gonzalo, se encontraron restos de un nido de carancho negro con un pollo volantón vivo y otro muerto. Tanto en el nido como en sus alrededores se detectaron restos de petrel azulado Halobaena caerulea (Gmelin, 1789).

El día 22 de diciembre de 2010, se encontró un nido de carancho negro ubicado en una abrupta quebrada de difícil acceso (perpendicular a un acantilado), bajo una grieta rocosa a 15 m.s.n.m. y distante a unos $40 \mathrm{~m}$ de la playa, en el margen norte de isla Gonzalo. El nido estaba ocupado por un polluelo (Fig.

Tabla 1. Preguntas que guiaron las entrevistas al personal del Faro Diego Ramírez, sur de Chile.
1) ¿Cuántos caranchos negros viven en isla Gonzalo?
2) ¿Los vé durante todo el año?
3) ¿De qué se alimentan?
4) ¿Ha visto algún nido?
5) ¿Qué impresión tiene de esta ave?
6) ¿Existe alguna amenaza para la conservación de esta ave?

1) de crecimiento avanzado (peso $1680 \mathrm{~g}$, longitud del tarso derecho $93,8 \mathrm{~mm}$, longitud y profundidad del pico 28,1 y $21,4 \mathrm{~mm}$, respectivamente). Esta nidada se encontraba vigilada por uno de sus progenitores, que mediante agudos y cortos gritos atrajo la presencia de su pareja. El nido estaba compuesto por hojas de coirón Poa flabellata [(Lam.) Raspail], con un diámetro de $35 \mathrm{~cm}$ y profundidad máxima de copa de $6 \mathrm{~cm}$. Alrededor de este nido se registró la presencia de restos de aves depredadas, logrando identificar cuatro alas (dos izquierdas y dos derechas, con una longitud promedio de $21,1 \pm 0,7 \mathrm{~cm}$ ) y una pata (cuya longitud de tarso fue $2,6 \mathrm{~cm}$ ) de petrel azulado.

El día 07 de enero 2011 se registró la existencia de otro nido de carancho negro en un acantilado ubicado en la porción sureste de isla Gonzalo. La inaccesibilidad y pendiente del acantilado impidió a los investigadores llegar hasta el nido, pero la conducta (tipo de grito emitido por los caranchos y los movimientos desplegados por la pareja) y plumaje reproductivo de las aves confirmó su existencia.

Sumando observaciones sobre la alimentación del carancho negro en isla Gonzalo, el 29 de noviembre 2010 se registró a un individuo salir volando desde una colonia de pingüino penacho amarillo Eudyptes chrysocome (Forster, 1781), llevando un polluelo de esta última entre sus garras. Así también, fue común observar caranchos negros merodear en las colonias del pingüino de penacho amarillo, albatros de ceja negra Thalassarche melanophrys (Temminck, 1839) y albatros de cabeza gris T. chrysostoma (Foster, 1785) en isla Gonzalo.

De manera ocasional, en isla Gonzalo se observó a un individuo de skua Stercorarius chilensis (Bonaparte, 1857) atacar a un individuo de carancho negro, sin lograr espantarlo, desconociéndose la existencia de algún tipo de competencia entre estas aves de similar condición trófica.

Dentro de las conductas observadas sobre este rapaz en isla Gonzalo, destaca su confianza y curiosidad ante la presencia humana, así como también la colecta y robo de objetos (e.g., gorros y guantes) e instrumentos de pequeño tamaño (e.g., dispositivos portátiles).

En total se entrevistó a seis fareros de isla Gonzalo, quienes indicaron apreciar un aumento en la abundancia del carancho negro, durante los últimos años. Obteniendo un promedio de $17 \pm 4$ individuos presentes en la isla (primavera 2012), con 


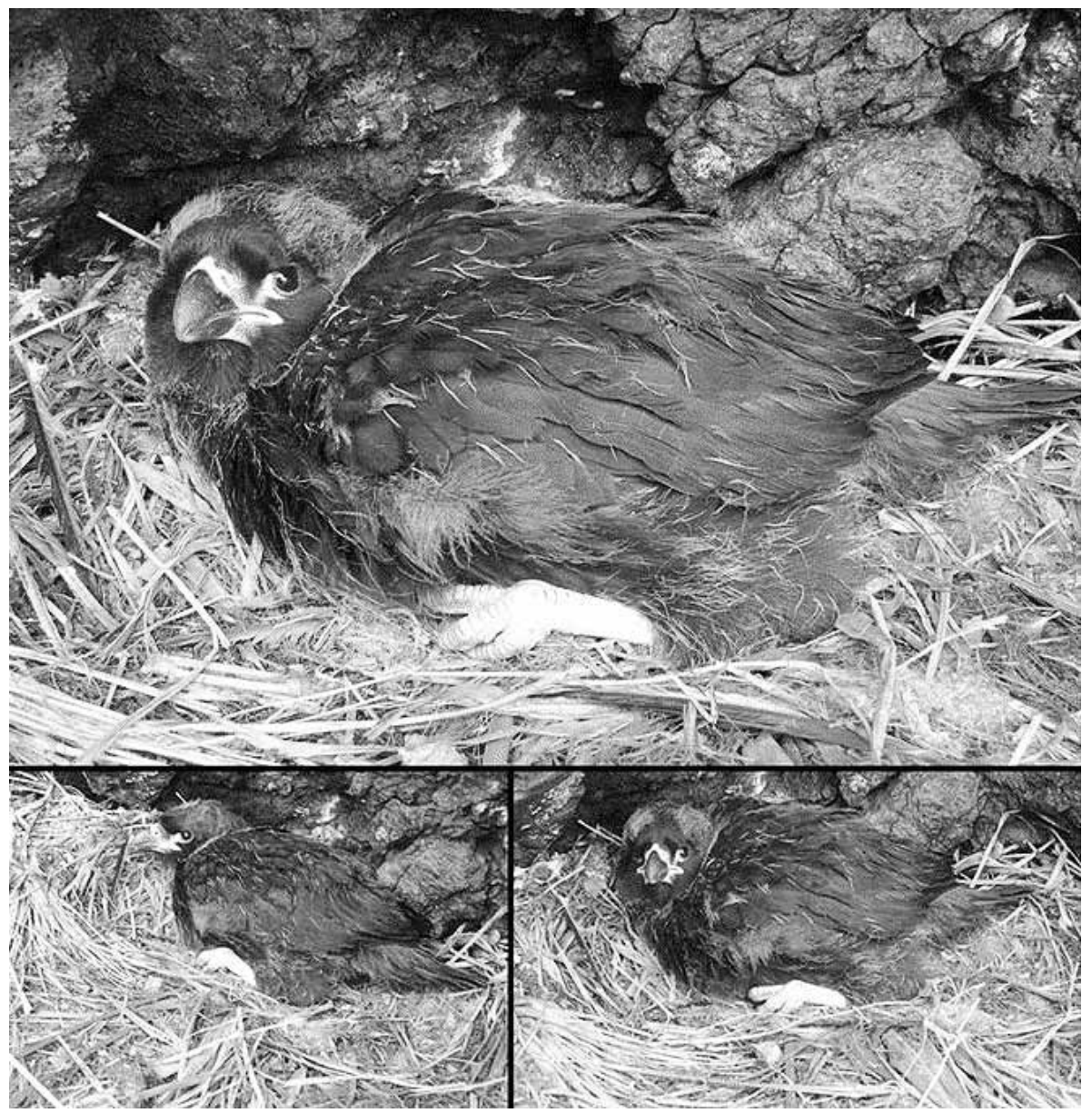

Fig. 1. Polluelo de carancho negro Phalcoboenus australis en un nido encontrado en isla Gonzalo, archipiélago Diego Ramírez, Chile. Fotografiado 26/12/2010.

presencia durante todo el año. Este rapaz se alimenta de organismos varados en la playa (macroalgas, moluscos, restos de aves y mamíferos marinos), así como también de los restos de alimentos emitidos por los fareros como grasa, carne, pan, arroz y fideos, indicando observar una mayor presencia de esta ave alrededor de la casa durante la temporada invernal (período en que la isla está cubierta por nieve). Reconociendo $(n=6)$ que pese a destinar esfuerzos de búsqueda, nunca han logrado encontrar un nido de carancho negro. Esta ave es curiosa y confiada, siempre atenta a las actividades humanas, existiendo casos $(n=5)$ en que los caranchos robaron materiales de trabajo de los fareros como brochas para pintar, martillos, hasta un equipo de radio. Con respecto a la impresión personal sobre este rapaz, la totalidad indica que en un comienzo les generó molestias por daños instrumentales (i.e., romper cables y antenas) que con el pasar del tiempo lograron apreciar su servicio ambiental (consumo de cadáveres como vigilancia sanitaria), así como también parecerles visualmente atractivo y entretenido. Sin identificar $(n=6)$ amenaza alguna para la conservación del carancho negro en isla Gonzalo.

La información publicada sobre la abundancia del carancho negro en isla Gonzalo, indica que la población tuvo un decrecimiento temporal desde estabilizada con 15 individuos durante el verano de los años 1981 y 1989, hasta su completa ausencia en el verano 2006 (Wallace 1991, Schlatter \& Riveros 1997, Marín et al. 2006). En la actualidad, el carancho negro presenta una recuperación de su población en isla Gonzalo, desconociéndose las causas de su pasado decrecimiento. 
Con respecto a la alimentación del carancho negro, se ha descrito que aves marinas como el petrel azulado y el pingüino penacho amarillo conforman la dieta de este rapaz, tanto para pasadas observaciones en isla Gonzalo, así como también en isla de los Estados (5445'S; 64²0'O), Argentina (Wallace 1991, Schiavini et al. 1999). En islas Malvinas (51ㄴㄱ' ${ }^{\circ}$; $61^{\circ} 18^{\prime} \mathrm{O}$ ), la principal presa registrada para el carancho negro fue el petrel paloma de pico delgado Pachyptila belcheri (Mathews, 1912) (Catry et al. 2008), cuyo peso corporal fue similar al del petrel azulado en isla Gonzalo [Peso promedio: $168,2 \mathrm{~g}$ (con rangos de 134,6 a $240 \mathrm{~g}$ ); $\mathrm{n}=54$; ambos sexos durante verano 2011 (CG Suazo \& JA Cursach, datos no publicados)].

Junto a isla Noir, el presente reporte conforma a isla Gonzalo como el segundo sitio de nidificación descrito para el carancho negro en Chile, que en función a los registros de sobrevivencia de nidada [2 a 3 polluelos por nido (Marín et al. 2006, Catry et al. 2008)] descritos para esta rapaz, indican de forma preliminar un bajo éxito reproductivo del carancho negro en isla Gonzalo. Lo anterior, evidencia y fundamenta la necesidad de aumentar esfuerzos humanos y económicos para la investigación en islas subantárticas del sur de Chile y de forma específica en el archipiélago Diego Ramírez.

\section{AGRADECIMIENTOS}

A la Universidad de Los Lagos por financiar los costos de esta publicación. A la III Zona Naval de la Armada de Chile. A la colaboración de Jaime Ojeda, Víctor Raimilla y Sebastián Rosenfeld. Al Dr. Adrián Schiavini por facilitar literatura. Al financiamiento de CIEP, CONICYT, Beca Nibaldo Bahamonde (U. de Los Lagos) y Pacific Seabird Group.

\section{LITERATURA CITADA}

BirdLife International. 2012. Factsheet Especie: Phalcoboenus australis. Descargado de http:// www.birdlife.org el 17/10/2012.

Catry, P., M. Lecoq \& I.J. Strange 2008. Population growth and density, diet and breeding success of striated caracaras Phalcoboenus australis on New Island, Falkland Islands. Polar Biology, 31: 1167-1174.

Cursach, J.A., C.G. Suazo, C.N. Tobar \& J.R. Rau 2012. Biometría del churrete austral Cinclodes antarcticus en isla Gonzalo, archipiélago Diego Ramírez, sur de Chile. Gayana, 76: 50-52

Frere, E., A. Travaini, A. Parera \& A. Schiavini 1999. Striated Caracara (Phalcoboenus australis) population at Staten and Año Nuevo Islands. Journal of Raptor Research, 33: 268-269.

Marín, M., A. Kusch, D. Oehler \& S. Drieschman 2006. Distribution, breeding and status of the striated caracara Phalcoboenus australis (Gmelin, 1788) in southern Chile. Anales Instituto de la Patagonia, 34: 65-74

Pincheira-Ulbrich, J., J. Rodas-Trejo, V. Almanza \& J.R. Rau 2008. Estado de conservación de las aves rapaces de Chile. Hornero, 23: 5-13

Schlatter, R.P. \& G.M. Riveros 1997. Historia Natural del Archipiélago Diego Ramírez, Chile. Serie Científica INACH, 47: 87-112

Schiavini, A., E. Frere, P. Yorio \& A. Parera 1999. Las aves marinas de la isla de los Estados, Tierra del Fuego, Argentina: Revisión histórica, estado poblacional y problemas de conservación. Anales Instituto de la Patagonia, 27: 25-40.

Wallace, G.E. 1991. Noteworthy birds records from southernmost Chile. Condor, 93: 175-176. 